Benno Kirsch

Private Sicherheitsdienste im öffentlichen Raum 
Benno Kirsch

\section{Private Sicherheitsdienste im öffentlichen Raum}

Formen und Folgen der Zusammenarbeit mit der Polizei in Berlin und Frankfurt am Main

Westdeutscher Verlag 
Bibliografische Information Der Deutschen Bibliothek

Die Deutsche Bibliothek verzeichnet diese Publikation in der Deutschen Nationalbibliografie; detaillierte bibliografische Daten sind im Internet über $<$ http://dnb.ddb.de $>$ abrufbar.

D 188

\section{Auflage Mai 2003}

Alle Rechte vorbchalten

(1) Westdeutscher Verlag/GWV Fachverlage GmbH, Wiesbaden 2003

Lektorat: Nadine Kinne

Der Westdeutsche Verlag ist ein Unternehmen der Fachverlagsgruppe BertelsmannSpringer. www.westdeutscher-verlag.de

Das Werk einschließlich aller seiner Teile ist urheberrechtlich geschützt. Jede Verwertung außerhalb der engen Grenzen des Urheberrechtsgesetzes ist ohne Zustimmung des Verlags unzulässig und strafbar. Das gilt insbesondere für Vervielfältigungen, Übersetzungen, Mikroverfilmungen und die Einspeicherung und Verarbeitung in elektronischen Systemen.

Die Wiedergabe von Gebrauchsnamen, Handelsnamen, Warenbezeichnungen usw. in diesem Werk berechtigt auch ohne besondere Kennzeichnung nicht zu der Annahme, dass solche Namen im Sinne der Warenzeichen- und Markenschutz-Gesetzgebung als frei zu betrachten wären und daher von jedermann benutzt werden dürften.

Umschlaggestaltung: Horst Dieter Bürkle, Darmstadt

Gedruckt auf säurefreiem und chlorfrei gebleichtem Papier

ISBN 978-3-531-14009-4 ISBN 978-3-322-90734-9 (eBook)

DOI 10.1007/978-3-322-90734-9 


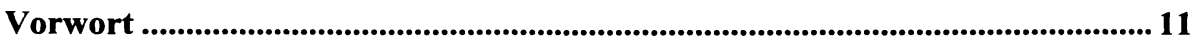

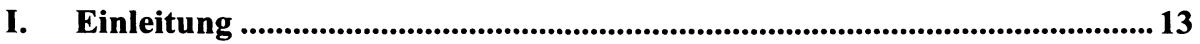

1. Das Verhältnis zwischen privaten Sicherheitsdiensten und der Polizei............ 13

1.1 Annäherung an ein Alltagsphänomen ................................................... 13

1.2 Im Zentrum der Aufmerksamkeit ...................................................... 14

1.3 Welche Folgen hat die Zusammenarbeit? ................................................. 16

2. Demokratie, Öffentlichkeit und bürgerliche Freiheit........................................ 17

2.1 Öffentlichkeit als zentrale demokratische Ressource ................................ 17

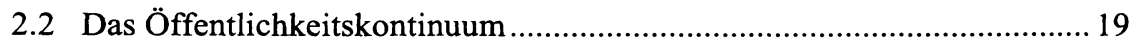

2.3 Verteidigung der Marktwirtschaft gegen ihre Fürsprecher .......................2 21

3. Das Feld und die Bedingungen für empirische Sozialforschung .......................24

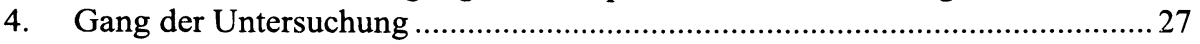

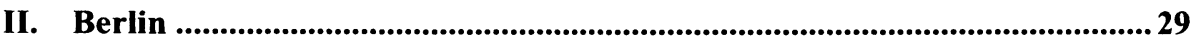

1. Nach dem Mauerfall: Armut überwindet die Teilung der Stadt .......................29

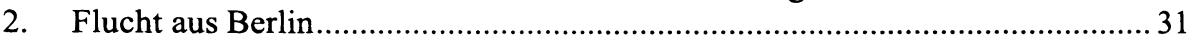

2.1 Verdrängung durch Aufwertung im Quartier Pulvermühle ........................ 31

2.2 Das trügerische Idyll von Neu Karow …………...................................... 34

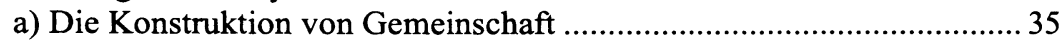

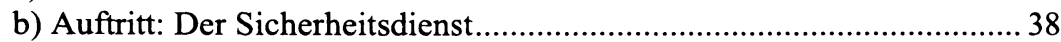

c) Zusammenfassende Bewertung ............................................................... 43

2.3 „Daimler City, „Sony Center“ und andere shopping malls........................ 44

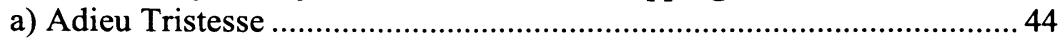

b) ,Jederzeit freundlich, hell, sauber, sicher“...........................................4 46

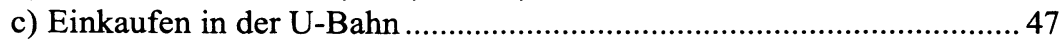

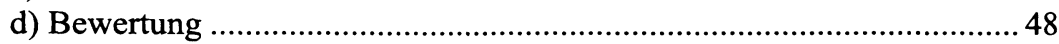

2.4 Weitere Varianten der Privatisierung öffentlichen Raums ....................... 49

2.5 Zwischenfazit: die Sicherheit von teil-öffentlichen Orten ........................51

3. Sicherheit in öffentlichen Verkehrsmitteln ................................................52

3.1 Das „Sicherheitskonzept für den ÖPNV“...............................................5

3.2 Sicherheit durch Personaleinsatz ............................................................ 56

3.3 Wie die gemischten Streifen bei der BVG für Ordnung sorgen .................59

3.4 Externes Sicherheitspersonal bei der BVG .............................................. 61

3.5 U-Bahn-Sicherheit durch Technik und Sauberkeit ...................................62

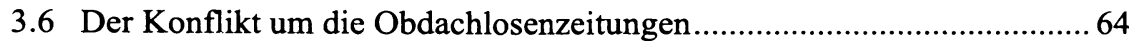

3.7 Zwischenfazit: Sicherheit durch Sauberkeit?.........................................67

4. Sicherheit, Sauberkeit und Kriminalität.....................................................70

4.1 Die Kampagne „Saubere Stadt Berlin“................................................. 70 
4.2 Armut und Sucht als polizeiliche Ordnungsprobleme ........................... 74

4.3 Die Umweltstreifen der Berliner Bezirksämter...................................... 75

4.4 Strategien der ,Sicherheit' und ihre Implikationen .................................79

5. Die Gewährleistung öffentlicher Sicherheit durch die Polizei........................ 81

5.1 Strategien und Befugnisse der Berliner Polizei ..................................... 82

5.2 Vorbereitungen für den Ausnahmezustand? ........................................8 87

6. Der Bundesgrenzschutz und die Deutsche Bahn AG.................................... 88

6.1 Der Bundesgrenzschutz: Geschichte und Struktur.................................. 88

6.2 Die Bundespolizei in Berlin............................................................ 90

6.3 Die durchaus intendierten Folgen des Wandels der Bundesbahn zum „Unternehmen Zukunft“ ........................................................... 90

6.4 Die Zusammenarbeit von BGS und Wachdienst .................................... 94

6.5 Zusammenfassende Bewertung ............................................................ 96

7. Police Private Partnership in der City West.............................................. 97

7.1 Der Niedergang des Kurfürstendamm ................................................. 97

7.2 Ein Sicherheitsdienst in den Straßen von Berlin....................................99

7.3 Der Senat zieht nach: eine Spezialeinheit gegen Hütchenspieler............ 100

7.4 Zusammenfassende Bewertung ........................................................... 102

8. Zwischenfazit: Es kommt auf den Begriff an ........................................... 103

III. Frankfurt am Main ....................................................................... 107

1. Der Landeplatz für spekulatives Kapital................................................. 107

2. Die Privatisierung von Öffentlichkeit.................................................... 109

3. Sicherheit in Frankfurts S- und U-Bahnen................................................. 112

3.1 Das Sicherheitsnetzwerk im ÖPNV ............................................... 112

3.2 Der kleine Betrug....................................................................... 114

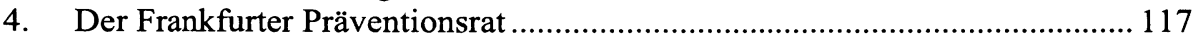

4.1 Präventionsarbeit mit Bürgerbeteiligung ......................................... 118

4.2 Problembewusstsein wecken ............................................................... 121

4.3 Und im Hintergrund die Polizei ............................................................ 123

5. Die Männer vom Ordnungsamt .......................................................... 124

5.1 Bürokratische Verortung der „Ganzheitssachbearbeiter“ “.................... 125

5.2 Ruhe und Ordnung in den Straßen von Frankfurt................................ 129

5.3 Die Stellung der HiPOs innerhalb des Gewaltmonopols ..................... 131

5.4 Die Ausdehnung kommunaler Sicherheitsstrukturen.......................... 134

6. Die Gewährleistung von Sicherheit durch die Polizei ................................... 135

6.1 Eine hochdifferenzierte Behörde ................................................... 136

6.2 Kooperation mit dem Bundesgrenzschutz ......................................... 141

6.3 Der Kooperationsvertrag zwischen Polizei und Bewachungsgewerbe ... 143

6.4 Informelle Zusammenarbeit im 1. Revier .............................................. 146

7. Zwischenfazit: ein engmaschiges Netz ............................................... 148

IV. Zwischenfazit: kommunale Kriminalpolitik ....................................... 151

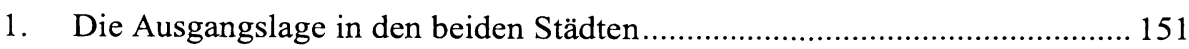

2. Die ordnungspolitische Rolle der Kommunen.......................................... 153

3. Die Risiken der kommunalen Sicherheitsstrategie .................................. 154 
1. Die empirische Erforschung der Lebenswelt von Wachleuten ...................... 156

1.1 Theorie und Praxis teilnehmender Beobachtung .................................. 156

a) Distanz und Empathie als modus operandi .................................... 157

b) On the beat ........................................................................... 158

1.2 Der Wachmann als Experte in Sachen Sicherheit................................. 159

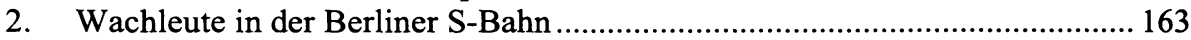

2.1 Monotonie, ein eingeschränktes Blickfeld und selektives Interesse ....... 163

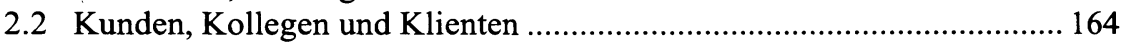

a) Das berufliche Netzwerk ............................................................ 164

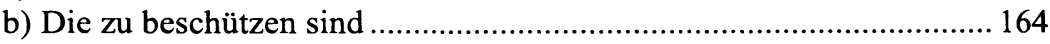

c) Unter Beobachtung ................................................................. 165

d) Unsichtbar und doch stets präsent: die Leitstelle........................... 166

e) Die ,Kollegen' von der Polizei ...................................................... 166

2.3 Dienstablauf und Arbeitsbedingungen............................................. 167

a) Einweisung und Dienstbeginn ............................................... 167

b) Zugbegleitung und -bewachung .............................................. 168

c) Dienstschluss, Kehrfahrten, Besonderheiten meiner Beobachtung .... 168

d) Reflexion am nächsten Morgen: ein ruhiges Wochenende................. 169

3. „Wenn die Oma mit dem Koffer kommt ..." -

Elemente bewachungsgewerblicher Tätigkeit ....................................... 170

3.1 Präsenz zeigen .................................................................... 170

3.2 Der Doppelcharakter der helfenden Dienstleistung ............................ 171

3.3 Facility Management ..................................................................... 172

3.4 Wahrnehmung des Hausrechts und Ordnungsmaßnahmen................... 173

a) Steter Tropfen höhlt den Stein .................................................... 175

b) Die Punker gehorchen ......................................................... 176

3.5 Die Fahrscheinkontrolle und ihre Folgen....................................... 177

3.6 Sicherung des Betriebsablaufs, Verhinderung von Personenschäden..... 181

a) Großeinsatz beim Fußballspiel ................................................. 181

b) Unbefugte auf den Gleisen ..................................................... 183

c) Ein Jugendlicher hat keinen Fahrschein ...................................... 184

d) Alltägliche Bagatellen ........................................................... 185

3.7 Der Krieg in den Kehren............................................................... 186

3.8 Zwischenfazit: das zum Teil eingelöste Versprechen ............................ 188

4. Konflikte und Gewalt gegen Wachleute .................................................... 189

5. Fehlverhalten von Wachleuten ................................................................ 192

5.1 Zwei gewaltsame Vorfälle ............................................................. 193

a) Die Misshandlung von „,Oz“ “................................................... 193

b) Ein Festgenommener wird abgeführt und verletzt sich selbst ........... 195

5.2 Wachleute als rassistische Gewalttäter? ........................................... 197

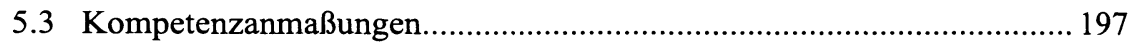

5.4 Unterlassene Hilfeleistung? ................................................................. 198

5.5 Todesgefahr durch Asphyxie ........................................................... 199

5.6 Zwischenfazit: Freispruch aus Mangel an Beweisen ........................... 200 
6. Zwischenfazit: Wachleute und Gewalt .....................................................200

6.1 Ziele, wie sie erreicht werden und welche Konflikte daraus entstehen ..200

6.2 Das Gewaltkontinuum ........................................................................ 202

6.3 Strukturelle Gewalt und individuelle Verantwortung ...............................205

VI. Integration in die Rechtsordnung .................................................................... 207

1. Die privatrechtlichen Grundlagen sicherheitsgewerblicher Tätigkeit.............207

1.1 Aus dem Eigentum abgeleitete Rechte ................................................207

1.2 Die Not- und Jedermannrechte stehen auch den Wachleuten zu .............208

1.3 Sanktionierung von Schwarzfahrten ................................................... 211

1.4 Bewertung: Ein Netz von Regelungen.......................................................2 213

2. Spezielle Normierung durch Gewerbeordnung und Gesetzesvollzug.............213

2.1 Der Zugang zum Bewachungsberuf.................................................... 213

2.2 Normierung der Berufsausübung in der Bewachungsverordnung ...........216

2.3 Auch die Berufsgenossenschaft nimmt Einfluss...................................... 217

2.4 Übersetzung für die Wachleute durch die Dienstanweisung .................. 218

2.5 Behördliche Kontrolle der Sicherheitsdienste........................................ 219

2.6 Bewertung: Defizite sind nicht zu erkennen ......................................... 222

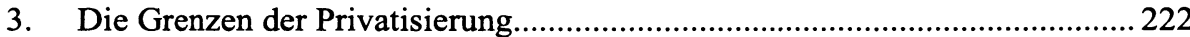

3.1 Eine „rechtsstaatlich saubere Kooperationsalternative“?.........................223

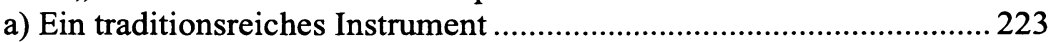

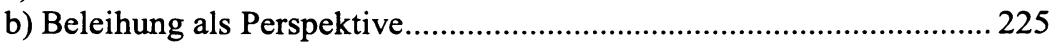

3.2 Die dritte Gewalt schreitet ein ............................................................ 226

a) Eine Kontrollstelle im Hessischen:

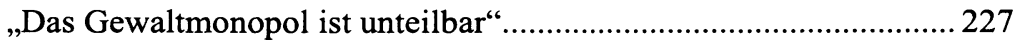

b) Parkraumbewirtschaftung in Berlin:

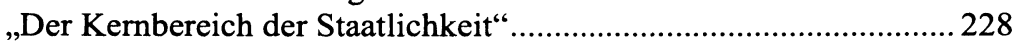

c) Die Reichweite des Gewaltmonopols ..............................................229

4. Die Diskussion über ein „Sicherheitsgewerbegesetz“..................................2230

4.1 Ansätze zu einer gesetzlichen Regelung ..............................................231

4.2 Bewertung: unklarer Zweck, ungute Folgen ....................................... 233

4.3 Verbrechensbekämpfung durch private Sicherheitsdienste?...................234

4.4 Nachtrag: die Änderung von 2002 …..................................................2236

5. Zwischenfazit: verschiedene Nicht-Zusammenhänge ……………...............238

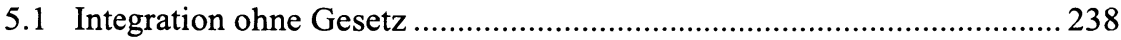

5.2 Die Stellung der Polizei bleibt erhalten ............................................... 240

VII. Das Bewachungsgewerbe im ,System Innerer Sicherheit' ......................... 242

1. Grundelemente sicherheitsgewerblicher Tätigkeit........................................242

1.1 Aufgaben und Tätigkeiten der Privaten ............................................. 242

1.2 Sicherheit ohne Bezug zu Kriminalität .................................................. 244

1.3 Von zentraler Bedeutung: hybride Formen von Öffentlichkeit .............. 244

1.4 Die Rolle der Kommunen ...................................................................... 246

2. Die Folgen des Einsatzes von Sicherheitsdiensten in dreifacher

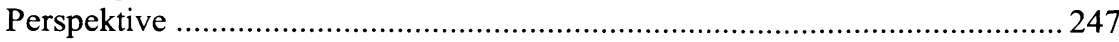

2.1 Polizei: Fortsetzung der präventiven Kehre ..........................................248 
2.2 Die Randgruppen bleiben police property ............................................. 250

2.3 Bürger: subtile Verhaltenssteuerung durch Kontrolle............................... 251

3. Gemeinsamer Grundkonsens privater und öffentlicher

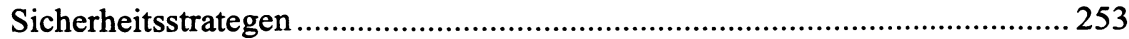

3.1 Unsicherheit als Konsequenz privater Sicherheitsgewährleistung.......... 253

3.2 Strategien der, Inneren Sicherheit' ........................................................... 255

4. Die Integration des Bewachungsgewerbes in das

,System Innerer Sicherheit' ............................................................................257

4.1 Ein weiterer Wachstumsring der Polizei.............................................. 257

4.2 Die Geschichte des Bewachungsgewerbes als Geschichte staatlicher

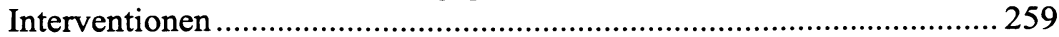

4.3 Die Zukunft des Nationalstaats im Angesicht der Privatisierung ............ 262

4.4 Zur Kommodifizierung von Sicherheit in Foucaultscher Perspektive .... 264

4.5 Die Folgen neoliberaler Regierungstechniken ...................................... 267

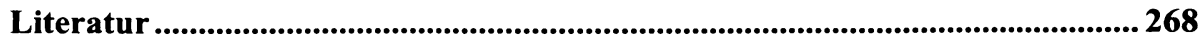




\section{Vorwort}

Die nachfolgende Arbeit wurde im Juni 2002 vom Fachbereich Politik- und Sozialwissenschaften der Freien Universität Berlin als Dissertation angenommen. Ich wäre nicht in der Lage gewesen, sie zu schreiben, wenn mir nicht zahlreiche Menschen in verschiedener Weise geholfen hätten. Mein herzlicher Dank gilt Herrn Prof. Dr. Wolf-Dieter Narr, der das Vorhaben von der ersten Ideenskizze bis zum fertigen Manuskript begleitete und mir immer wieder behutsam neue Wege wies. Ebenfalls bedanke ich mich bei Herrn Priv.-Doz. Dr. Norbert Pütter, der mir insbesondere bei der Konzeption des empirischen Teils zur Seite stand, und bei Frau Prof. Dr. Barbara Riedmüller, die das Zweitgutachten anfertigte.

Freunde haben mich in verschiedenen Phasen unterstützt, vor allem indem sie Passagen der Arbeit lasen und konstruktive Kritik übten. Zu erwähnen sind hier insbesondere Dipl.-Pol. Albrecht von Lucke, Dr. Hiltrud Wallenborn, Dipl.-Soz. Jutta Kirsch und Dr. Michael Haus. Meine Eltern haben nicht nur mein Studium großzügig finanziert, sondern meinen Weg auch immer ideell unterstützt. Von der KonradAdenauer-Siftung habe ich ein Stipendium erhalten, das mich in die komfortable Lage versetzte, mich ganz auf meine wissenschaftlichen Arbeit zu konzentrieren.

Was aber hätte ich machen können, wenn mir nicht die zahlreichen genannten und nicht genannten Experten aus Polizei, Bewachungsunternehmen, Lokalverwaltungen und sozialen Diensten für Gespräche und teilnehmende Beobachtungen zur Verfügung gestanden hätten? Nichts! -, denn das Objekt meiner wissenschaftlichen Begierde wäre in einem undurchdringlichen Nebel verschwunden, der alle Anstrengungen zum Verstehen unmöglich gemacht hätte. Ich bedanke mich deshalb auch bei ihnen, die meinem Vorhaben ausnahmslos aufgeschlossen und interessiert gegenüber standen und mir bereitwillig Einblick in eine fremde Welt gewährten.

Berlin, im März 2003

Benno Kirsch 